\title{
Smart RopEx - Vorhersage des Versagenszeitpunkts synthetischer geflochtener Seile durch integrierte textilbasierte Monitoringsysteme
}

\author{
Melanie Wipfler, Nina Laar, Stefan Jockenhövel, Thomas Gries \\ Institut für Textiltechnik der RWTH Aachen University, Aachen, Otto-Blumenthal-Str. 1
}

\section{Zusammenfassung}

Im Projekt Smart RopEx wird ein textilbasiertes, in ein Faserseil integriertes Monitoringsystem entwickelt, das eine Aussage über die Lebensdauer des betrachteten Seils liefert. Damit wird es möglich, eine Aussage über die verbleibende Restlebensdauer $\mathrm{zu}$ erhalten und so eine optimale Ausnutzung des Produktlebenszyklus zu erreichen. Ebenso wichtig ist der Sicherheitsaspekt: Faserseile werden in sicherheitsrelevanten Bereichen kaum eingesetzt, da langjährige Erfahrungen, wie sie für Drahtseile vorliegen, noch fehlen. Das Smart RopEx Monitoringsystem soll diese fehlende Erfahrung durch statistisch gesicherte Aussagen zur verbleibenden Lebensdauer wettmachen.

\section{Problemstellung}

Seile aus Chemiefasern sind in zahlreichen Anwendungen, wie zur Sicherung von Personen, als Hebe- und Transportmittel, zur Vertäuung von Fracht- und Personenschiffen, als Fallschirmleinen sowie in Freizeitanwendungen wie dem Bergsport und dem Segelsport etabliert. In den letzten Jahren gewinnen sie jedoch auch in vielen Anwendungsbereichen an Bedeutung, in denen bislang Drahtseile eingesetzt wurden. Dies ist bei Betrachtung ihrer Vorteile gegenüber den konventionellen Drahtseilen naheliegend. Seile aus Hochleistungsfasern haben eine etwa vier bis fünffach größere spezifische Festigkeit und ein etwa $30-35 \%$ niedrigeres spezifisches Gewicht als Drahtseile. Insbesondere in Anwendungen, in denen große Seillängen erforderlich sind, oder in denen die Seile dynamischen Prozessen unterliegen, ist der Einsatz synthetischer Seile sinnvoll. Ein Beispiel dafür sind Aufzugseile. Diese Seile erreichen mitunter eine Länge, die aufgrund des aufsummierten Eigengewichts bei der Verwendung von Stahl zum Versagen führen würde. Darüber hinaus sind die Chemiefaserseile sehr gut zu handhaben und ermöglichen geringere Biegeradien als vergleichbare Stahlseile. Ein ebenfalls wichtiger Punkt ist ihre Korrosionsfreiheit.

Somit können Faserseile die Drahtseile in vielen Anwendungsbereichen nicht nur ersetzen sondern übertreffen. Da sie jedoch teurer als Drahtseillösungen sind, müssen sie über die komplette Nutzungsdauer in Bezug auf die Wirtschaftlichkeit mit dem gegenwärtigen Stand der Technik Schritt halten können. Noch weitaus wichtiger ist der Sicherheitsaspekt. Für die seit langem eingesetzten Drahtseile existieren verschiedene Prüfmethoden, ebenso ist eine breite Erfahrungsbasis vorhanden, die den Einsatz der Seile weitestgehend sicher macht [1].

Um die Sicherheit der Chemiefaserseile zu gewährleisten, werden in regelmäßigen Abständen zeit- und kostenintensive Sichtkontrollen durch Sachkundige durchgeführt. Eine umfassende Sicherheit kann jedoch aufgrund der diskontinuierlichen Prüfung und des Fehlens einer objektiven Beurteilung nicht gewährleistet werden. Um dennoch eine ausreichende Sicherheit zu erreichen, werden die Seile deutlich überdimensioniert und prophylaktisch nach einer festgelegten Nutzungsdauer ausgetauscht. Dies erfolgt unabhängig vom tatsächlichen Verschleißzustand und führt zu hohem Ressourcenverbrauch und hohen Kosten. Hierdurch wird der Einsatz synthetischer Seile durch die mangelnde Erfahrung mit dem Langzeitverhalten dieser Produkte und den damit einhergehenden hohen Sicherheitsrisiken trotz ihrer großen Vorteile erschwert.

Das Institut für Textiltechnik der RWTH Aachen beschäftigt sich bereits seit 2004 mit der Fragestellung, wie die Sicherheit von Faserseilen erhöht werden kann. Das Projekt „Sensorseil“ untersuchte verschiedene Möglichkeiten, den Verschleiß textiler Seile oder Bänder durch die Integration von Sensoren zu erfassen [2, 3]. Dazu wurden verschiedene fadenförmige mechanische oder elektrische Sensorkonzepte entwickelt und 
erstmalig erprobt. Hierbei wurde das Konzept der dehnungsinduzierten Widerstandsänderung leitfähiger Garne als erfolgversprechendstes Konzept ermittelt.

Dieses Konzept wurde in einem weiteren Projekt, dem Projekt „Smart Rope“, für die vier Beispielanwendungen Fallschirmleine, Mooringleine, Aufzugseil und Sicherungsseil erprobt. Die grundsätzliche Funktionsweise soll anhand Bild 1 erläutert werden.

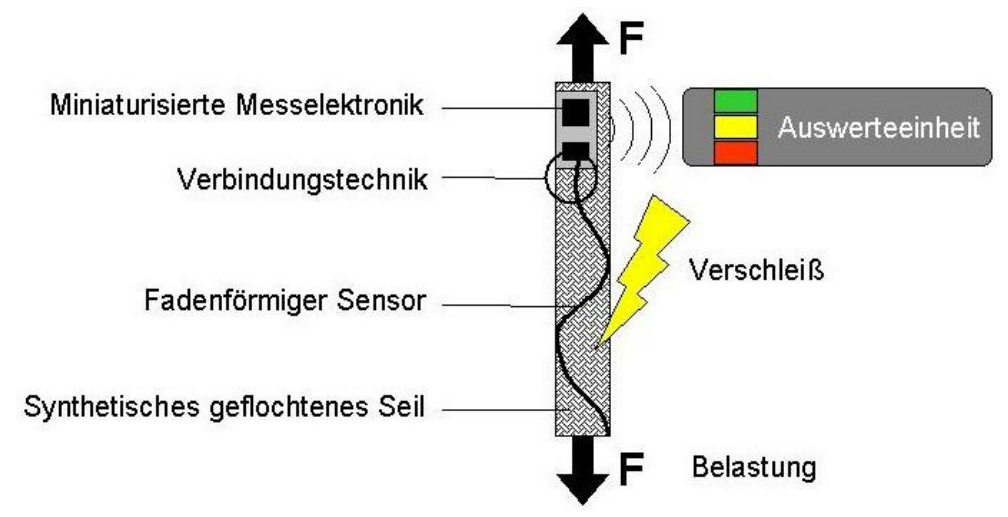

Bild 1 Komponenten des textilbasierten Monitoringsystems

In das zu überwachende geflochtene Seil wird ein fadenförmiger Sensor eingebracht. Die Integration erfolgt kontinuierlich im konventionellen Herstellungsprozess. Um die Eigenschaften des Seiles nicht zu verändern, darf durch den Sensor keine Veränderung der Seilkonstruktion erfolgen. Durch den Aufbau des textilen Sensors wird dies sichergestellt: Es handelt sich um ein polymeres Garn, das mit einer leitfähigen Schicht, vorzugsweise Silber, beschichtet wird. Diese Schicht verändert den textilen Charakter des Garns nicht. Durch die Dehnung des Seils und die dadurch erzielte Dehnung des Sensorfadens verändert dieser seinen Widerstand. Es handelt sich somit um einen textilen Dehnungsmessstreifen. Über eine geeignete Verbindungstechnik wird der Sensor mit der Messelektronik verbunden. Eine Auswerteeinheit zeigt den Zustand des Seils an [4, 5].

Das Ziel des Projektes „Smart Rope“, die Erhöhung der Funktionssicherheit geflochtener synthetischer Seile, konnte durch die Integration des Sensorsystems in die Faserseile erreicht werden [6]. So wurde es z.B. möglich, einen für ein Sicherungsseil durch die Norm EN 1891 - Kernmantelseile [7] vorgeschriebenen Abwurf mit definierter Last und aus definierter Höhe anzuzeigen. Das integrierte Monitoringsystem zeigt eindeutig an, dass das Seil mit einer Belastung, die äquivalent zum Absturz einer $80 \mathrm{~kg}$ schweren Person ist, belastet wurde.

Im Projektverlauf wurde als Defizit erkannt, dass die Verfügbarkeit objektiver Kriterien zur Beurteilung des Belastungs- und Verschleißzustands noch nicht ausreichend gegeben ist. Dies ist durch das Fehlen von Grenzwerten, die das tatsächliche Erreichen des Versagenszeitpunktes anzeigen, zu erklären. Zur Definition dieser Grenzwerte, die die Ablegereife anzeigen, ist ein erheblicher Versuchsaufwand notwendig. Die Ablegereife ist definiert als Zeitpunkt des rechtzeitigen Seiltausches vor Erreichen eines gefährlichen Zustandes, also der Unterschreitung eines erforderlichen Sicherheitsfaktors in Abhängigkeit von der Anwendung. Um die Forschungsergebnisse aus dem Projekt „Smart Rope“ weiter zu nutzen und sinnvoll zu vertiefen, erschien die Durchführung weiterer Schritte sinnvoll. Hierzu gehören

- die Erarbeitung von Grenzwerten, um die Ablegereife von Seilen anzeigen zu können

- die Weiterentwicklung der entwickelten Sensorkomponenten zur Anzeige dieser Grenzwerte

- die Kombination beider Bestandteile zu funktionsfähigen Gesamtsystemen.

Das Beispiel des Sicherheitsseil verdeutlicht die Problematik: Mithilfe des integrierten Monitoringsystems ist es möglich, einen normgerechten Belastungsfall, also einen Abwurf, anzuzeigen. Das Monitoringsystem lässt jedoch die Frage offen, wie viele weitere lastäquivalente Abwürfe das Seil noch ertragen kann ohne zu versagen. Die Norm EN 1891 fordert fünf weitere Abwürfe aus zwei Meter Höhe. Diese Forderung ist durch jedes normgerecht hergestellte Seil zu erfüllen und stellt die konstruktive Sicherheit dar, um den ersten Abwurf in jedem Fall sicher zu ertragen. Um dies zu erreichen, werden die Seile überdimensioniert. Sie sind also in der Lage, auch weitere Abwürfe zu überstehen. Da jedoch nicht genau vorhergesagt werden kann, wie viele Abwürfe ein Seil tatsächlich erträgt, werden die Seile frühzeitig, bereits nach einem Sturz, ausgetauscht. Diese grundsätzliche Frage nach der sogenannten Ablegereife der Chemiefaserseile ist also noch offen. Einen Ansatz zur Beantwortung dieser Frage soll das Projekt „Smart RopEx“ liefern, in dem die Erforschung 
der Ablegereife synthetischer geflochtener Seile und die entsprechende Entwicklung von Sensoren, Systemen und Algorithmen das Thema ist.

\section{Lösungsansatz}

Im Projekt „Smart RopEx“ werden die drei Beispielanwendungen Aufzugseil, Hebeschlinge und Windenseil betrachtet. Im Gegensatz zu den in den vorherigen Projekten betrachteten Anwendungen bewegen sich die drei Anwendungen nicht nur in einem äußerst sicherheitssensiblen Bereich, sondern zusätzlich im hochpreisigen Segment. Dies verändert die Kostenstruktur und erfordert eine möglichst optimale Ausnutzung des Produktlebenszyklus unter Einhaltung hoher Sicherheitsstandards.

Anhand folgender Schritte und Teilziele werden die Systeme aufgebaut:

- Aufstellung eines statistischen und physikalischen Versagensmodells und Ermittlung der zugehörigen Kenngrößen zur Verschleißanzeige

- Entwicklung geeigneter Sensormodule ${ }^{1}$ zur Erfassung der Kenngrößen

- Integration der Sensormodule in die jeweilige Seilanwendung

- Entwicklung einer Verbindungstechnologie zur Anbindung der integrierten Sensormodule an die Elektronik

- Entwicklung eines Mess- und Auswertegeräts zur Auswertung der Kenngrößen und Anzeige der Restlebensdauer

Das Bild 2 stellt alle Komponenten zu einem Gesamtüberblick zusammen. Das grundlegende Prinzip bleibt wie im Projekt Smart Rope erhalten. Zur Anzeige der Ablegereife, bedingt durch die Auswirkungen von Zugbelastungen, Biegewechselbelastungen und Beschädigungen, aber auch sonstiger klimatischer Einflüsse wie Temperatur und Feuchtigkeit soll eine objektive und messbare Skala erarbeitet werden. Bisher existieren

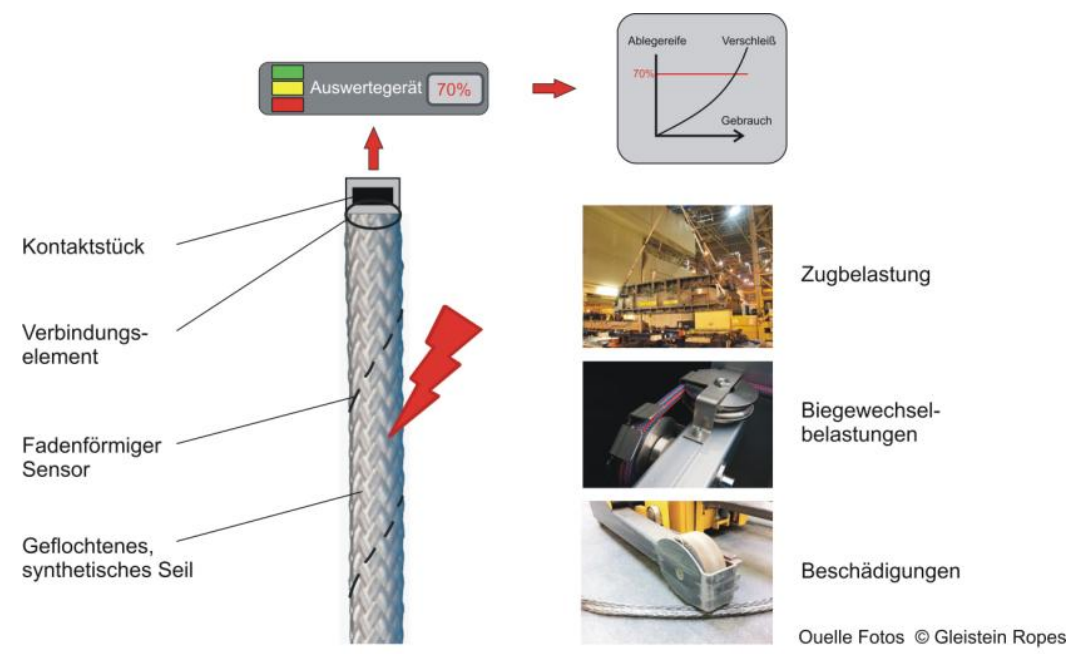

Bild 2 Erweiterte Prinzipskizze des textilbasierten Monitoringsystems

für Faserseile nur subjektive, durch erfahrene Gutachter zu bewertende, Ablegekriterien [8]. Das aufzustellende Versagensmodell soll sowohl diese Kenngrößen als auch Lebensdauerstatistiken des jeweiligen Seils beinhalten und stellt diese in einen Zusammenhang $\mathrm{zu}$ den Messwerten des Monitoringsystems. Somit kann die Ablegereife über der Gebrauchsdauer aufgetragen werden. Bei Erreichen eines im Projekt zu definierenden Verschleiß-Grenzwertes (als Beispiel in Bild 2 bei 70\% aufgetragen) wird der Nutzer informiert, um das Seil effizient und ökonomisch auszutauschen. Bei den betrachteten hochpreisigen Anwendungen stellt dies eine optimale Kosten-Nutzen-Relation bei ausreichend hohem Sicherheitsfaktor sicher.

\footnotetext{
${ }^{1}$ Sensormodul = Garn, Zwirn, oder anderes faden- oder bandförmiges, textiles Gebilde zur textilen Widerstandsmessung, das textiltechnisch so weiterverarbeitet worden ist, dass es Umwelt- und Verarbeitungseinflüssen stand hält.
} 
Danksagung:

Wir danken der Forschungsvereinigung Forschungskuratorium Textil e.V. für die finanzielle Förderung des Forschungsvorhabens AiF-Nr. 14107 BG/5, das im Programm zur Förderung der „Industriellen Gemeinschaftsforschung (IGF)“ aus Haushaltsmitteln des Bundesministeriums für Wirtschaft und Technologie (BMWi) über die Arbeitsgemeinschaft industrieller Forschungsvereinigungen e.V. (AiF) erfolgte.

Wir danken dem Bundesministerium für Bildung und Forschung sowie der VDI/VDE Innovation + Technik GmbH für die Förderung der Projekte „Smart Rope - Erhöhung der Funktionssicherheit von synthetischen geflochtenen Seilen und Leinen durch ein integriertes textilbasiertes Monitoringsystem“ (Verbundnummer 16SV3469) und „Smart RopEx - Anzeige des Versagenszeitpunktes synthetischer geflochtener Seile durch integrierte textilbasierte Monitoringsysteme“ (Verbundnummer W4TEX003).

Wir danken den Projektpartnern für die gute Zusammenarbeit in den Projekten Smart Rope und Smart RopEx: August Herzog Maschinenfabrik Gmbh \& Co. KG, Oldenburg; Barthels-Feldhoff KG, Wuppertal; Brüggemann $\mathrm{GmbH} \& \mathrm{Co}$. KG, Herdecke; Geo. Gleistein GmbH, Bremen; Gimpel IngenieurGesellschaft mbH, Aachen; Lehrstuhl Konstruktion und Entwicklung von Mikrosystemen (KE $\mu$ ), RWTH Aachen; SBU GmbH, Waldheim; Statex GmbH, Bremen; ThyssenKrupp GmbH, Neuhausen

\section{Literatur}

[1] Feyrer, K.: Drahtseile - Bemessung, Betrieb, Sicherheit, 2.Auflage, Berlin: Springer 2000

[2] Scheibner, W.; Thurner, F.; Möhring, U.; Stüve, J.; Janicijevic, D.; Gries, T. Sensorfäden für Belastung und Verschleiß von lastaufnehmenden Bändern und Seilen Band- und Flechtindustrie 43 (2006), H. 2, S. 30-35

[3] Möhring, U.; Scheibner, W.; Gries, T.; Stüve, J. Erhöhung der Funktionssicherheit von gewebten und geflochtenen lastaufnehmenden Bändern und Seilen für industrielle Anwendungen und Extremsportbereiche durch Integration von Sensoren für die Belastungs- und Verschleißkontrolle

Abschlussbericht zum Forschungsvorhaben AiF 14107 BG/5 am Textilforschungsinstitut ThüringenVogtland e.V. Greiz und am Institut für Textiltechnik der RWTH Aachen, Greiz Aachen 2006

[4] Giannikopoulos, T.; Stüve, J., Wipfler, M.; Gries, T.; Schomburg, W.K.: Micro Sensors for wear measurements in ropes; Proc. 20th MicroMechanics Europe Workshop, MME 2009 in Toulouse, Frankreich (2009) A05

[5] Wipfler, M.; Stüve, J.; Gries, T.: Smart Rope: Improved safety of braided synthetic ropes due to integrated threadlike sensors; Dörfel, A. (Ed.): Proceedings of the 2nd Aachen-Dresden International Textile Conference, Dresden, December 04-05, 2008. - Dresden : Institute of Textile and Clothing Technology, TU Dresden, 2008, Paper: p65_wipfler.pdf

[6] Stüve, J.; Wipfler, M.; Gries, T.

Smart Rope : Erhöhung der Funktionssicherheit von synthetisch geflochtenen Seilen und Leinen durch ein integriertes textilbasiertes Monitoringsystem

Abschlussbericht zum Verbundprojekt im Rahmenprogramm "Mikrosysteme 2004 - 2009" (VDI/VDE/IT 16SV3469) am Institut für Textiltechnik der RWTH Aachen University, Herzog Maschinenfabrik GmbH, ThyssenKrupp GmbH, Barthels-Feldhoff KG, Brüggemann GmbH + Co, Geo. Gleistein GmbH, Gimpel Ing.-GmbH, SBU GmbH, Aachen 2010

[7] $\mathrm{NN}$

DIN EN 1891 - Persönliche Schutzausrüstung zur Verhinderung von Abstürzen - Kernmantelseile mit geringer Dehnung; Deutsche Fassung EN 1891:1998

[8] Berufsgenossenschaftliche Regeln für Sicherheit und Gesundheit bei der Arbeit; BGR 152 - Gebrauch von Anschlag-Faserseilen 